\title{
Evidence of Large Polarons in Photoemission Band Mapping of the Perovskite Semiconductor $\mathrm{CsPbBr}_{3}$
}

\author{
M. Puppin $\odot,{ }^{1, *}$ S. Polishchuk $\odot,{ }^{1}$ N. Colonna $\odot,{ }^{2}$ A. Crepaldi $\odot,{ }^{3}$ D. N. Dirin $\odot,{ }^{4,5}$ O. Nazarenko, ${ }^{4,5}$ \\ R. De Gennaro $\odot,{ }^{2}$ G. Gatti, ${ }^{3}$ S. Roth, ${ }^{3}$ T. Barillot, ${ }^{1}$ L. Poletto, ${ }^{6}$ R. P. Xian $\odot,{ }^{7}$ L. Rettig $\odot,{ }^{7}$ M. Wolf, ${ }^{7}$ \\ R. Ernstorfer® ${ }^{7}$ M. V. Kovalenko, ${ }^{4,5}$ N. Marzari, ${ }^{2}$ M. Grioni $\odot,{ }^{3}$ and M. Chergui $\oplus^{1, *}$ \\ ${ }^{1}$ Laboratoire de Spectroscopie Ultrarapide and Lausanne Centre for Ultrafast Science (LACUS), \\ École Polytechnique Fédérale de Lausanne, ISIC, Station 6, CH-1015 Lausanne, Switzerland \\ ${ }^{2}$ Theory and Simulations of Materials (THEOS), and National Centre for Computational Design \\ and Discovery of Novel Materials (MARVEL), École Polytechnique Fédérale de Lausanne, CH-1015 Lausanne, Switzerland \\ ${ }^{3}$ Institute of Physics and Lausanne Centre for Ultrafast Science (LACUS), École Polytechnique Fédérale de Lausanne, \\ CH-1015 Lausanne, Switzerland \\ ${ }^{4}$ Laboratory of Inorganic Chemistry, Department of Chemistry and Applied Biosciences, \\ ETH Zürich, CH-8093 Zürich, Switzerland \\ ${ }^{5}$ Laboratory for Thin Films and Photovoltaics, EMPA Swiss Federal Laboratories for Materials Science and Technology, \\ Überlandstrasse 129, CH-8600 Dübendorf, Switzerland \\ ${ }^{6}$ National Research Council of Italy-Institute of Photonics and Nanotechnologies (CNR-IFN), \\ via Trasea 7, 35131 Padova, Italy \\ ${ }^{7}$ Fritz-Haber-Institut der Max-Planck-Gesellschaft, Faradayweg 4-6, 14195 Berlin, Germany
}

(Received 2 September 2019; revised manuscript received 30 March 2020; accepted 14 April 2020; published 20 May 2020)

Lead-halide perovskite (LHP) semiconductors are emergent optoelectronic materials with outstanding transport properties which are not yet fully understood. We find signatures of large polaron formation in the electronic structure of the inorganic LHP CsPbBr 3 by means of angle-resolved photoelectron spectroscopy. The experimental valence band dispersion shows a hole effective mass of $0.26 \pm 0.02 m_{e}, 50 \%$ heavier than the bare mass $m_{0}=0.17 m_{e}$ predicted by density functional theory. Calculations of the electron-phonon coupling indicate that phonon dressing of the carriers mainly occurs via distortions of the $\mathrm{Pb}-\mathrm{Br}$ bond with a Fröhlich coupling parameter $\alpha=1.81$. A good agreement with our experimental data is obtained within the Feynman polaron model, validating a viable theoretical method to predict the carrier effective mass of LHPs $a b$ initio.

DOI: 10.1103/PhysRevLett.124.206402

Hybrid organic-inorganic and inorganic lead-halide perovskites (LHPs) rival conventional semiconductors in multiple optoelectronic applications. LHP-based solar cells have established energy conversion efficiencies approaching 25\% [1]; light-emitting devices [2] and lasers [3] are gaining considerable interest thanks to high luminescence quantum efficiency [4]. The carrier diffusion length is exceptionally long in LHPs, reaching up to several micrometers $[5,6]$. This property results from the long carrier lifetimes, rather than from the carrier mobility [7]. While theory predicts small effective masses [8-13] $\left(\approx 0.1-0.3 m_{e}\right.$, where $m_{e}$ is the free electron mass), the reported mobilities are orders of magnitude lower than in conventional inorganic semiconductors $[7,14]$. The microscopic mechanism

Published by the American Physical Society under the terms of the Creative Commons Attribution 4.0 International license. Further distribution of this work must maintain attribution to the author(s) and the published article's title, journal citation, and DOI. underlying this unusual combination of transport properties is possibly the interplay between carriers and the ionic perovskite lattice $[7,15]$. In a polar crystal, longitudinaloptical (LO) phonon modes have a sizable long-range interaction with charge carriers, resulting in the formation of so-called Fröhlich polarons [16]. The polaron, heavier than a bare carrier, has a reduced mobility, compatible with the observed transport properties [15,17]. In particular, the screening of the Coulomb potential is modified in the case of polarons, purportedly explaining the observed carrier lifetimes $[17,18]$.

The optical properties of different LHPs are known to critically depend on the details of the lead-halide bond angles [19], highlighting the importance of carrier-lattice coupling in the photophysics of LHPs. The presence of polaron quasiparticles was indeed already proposed to model the results of several optical studies $[17,18,20]$.

In this Letter we report on experimental evidence of polaron formation by measuring its fingerprint in the electronic structure. We concentrate on the prototypical 
inorganic LHP CsPbBr 3 , which has lately attracted interest for applications, due to better thermal and radiation stability compared to hybrid organic-inorganic LHPs [21-25]. The momentum-resolved electronic structure of $\mathrm{CsPbBr}_{3}$ is determined by angle-resolved photoelectron spectroscopy (ARPES) and compared with $a b$ initio density functional theory (DFT). Our ARPES data provide a direct measurement of the hole effective mass $\left(m_{\text {exp }}\right)$ in $\mathrm{CsPbBr}_{3}$. The experiment reveals a mass enhancement of $50 \%$ compared to theory, which we attribute to electron-phonon coupling. $A b$ initio simulations of electron-phonon interaction show that $\mathrm{Pb}-\mathrm{Br}$ stretching modes dominate the interaction. Furthermore, our calculations provide a Fröhlich coupling parameter $\alpha=1.81$, indicating that carriers form large polarons, and predict a mass renormalization in good agreement with experimental data.

At room temperature $\mathrm{CsPbBr}_{3}$ assumes an orthorhombic lattice structure, which departs from the ideal cubic perovskite structure. The transition to the orthorhombic phase occurs below $360 \mathrm{~K}$ by rotation of the $\left[\mathrm{PbBr}_{6}\right]^{4-}$ octahedra surrounding the $\mathrm{Cs}^{+}$cation [26]. The orthorhombic structure is compared to an undistorted cubic one in Fig. 1(b), showing its larger real-space primitive cell and the octahedra's canting angle of approximately $10^{\circ}$ [14].

High-quality single crystals of $\mathrm{CsPbBr}_{3}$ were grown from liquid solution using an inverse temperature crystallization method [27]. The $\mathrm{CsPbBr}_{3}$ crystals were cleaved in situ under ultrahigh vacuum conditions. ARPES experiments were performed using extreme ultraviolet radiation from a high-harmonic laser source with a tunable photon energy between 20 and $40 \mathrm{eV}$ [28,29]. All data were collected at room temperature, in the orthorhombic phase of $\mathrm{CsPbBr}_{3}$, as confirmed by x-ray diffraction [30-52]. To rationalize the experimental results, we perform ab initio calculations using the Quantum ESPRESSO distribution $[53,54]$. The electronic structure was obtained at the generalized Kohn-Sham level using the hybrid functional scheme proposed by Heyd, Scuseria, and Ernzerhof [55,56] (HSE) for the exchange and correlation energy functional. The electron-phonon interaction was accounted for within the Fröhlich model [57] with parameters obtained averaging the $a b$ initio Fröhlich vertex [58,59]. Further details concerning the experimental methods and the DFT calculations are given in the Supplemental Material [30].

The valence band (VB) photoemission intensity distribution is plotted as a function of energy and in-plane momentum wave vectors in Fig. 1(d), for a photon energy of $37 \mathrm{eV}$. The left half of Figs. 1(e) and 1(f) shows two cuts at constant energy of the three-dimensional ARPES intensity distribution, at the valence band maximum (VBM) and $1.1 \mathrm{eV}$ below the VBM. The energy zero was set at the VBM, determined from the energy of the peak maximum.

Four valence band maxima are clearly resolved at the four corners $(\bar{M})$ of the surface-projected Brillouin zone (SBZ), following the periodicity expected for the cubic phase of the system, despite the structural phase transition (a)

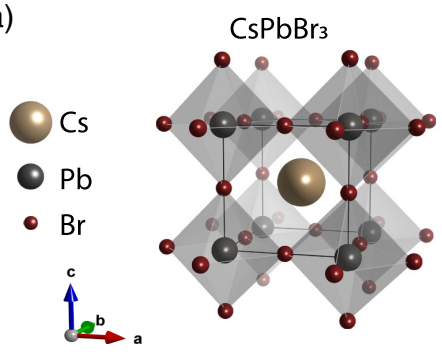

(b)

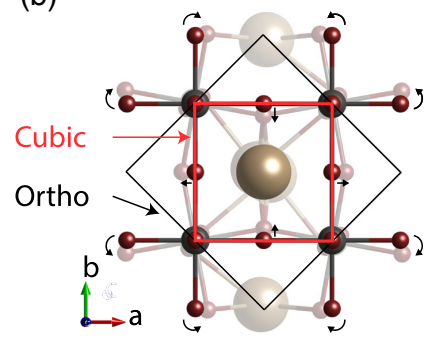

(c)

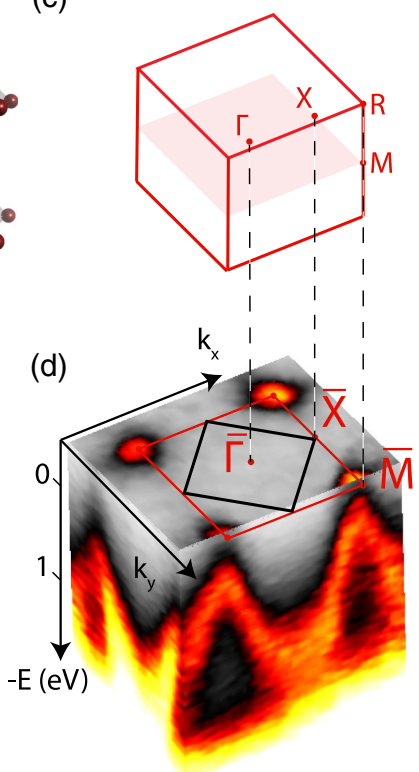

(e)

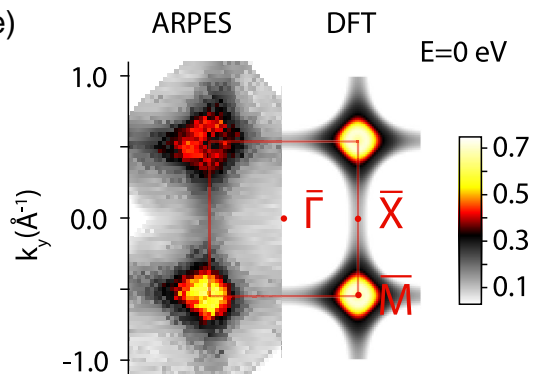

(f)

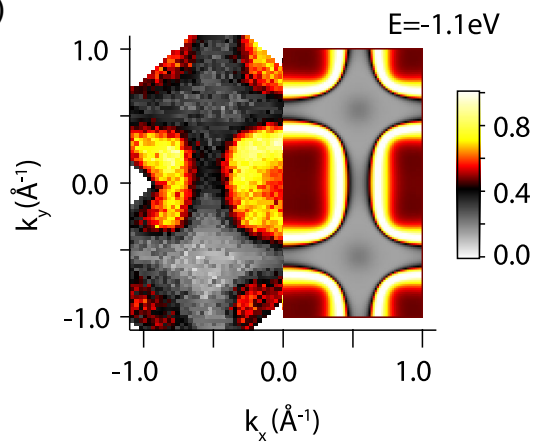

FIG. 1. Schematic structure of $\mathrm{CsPbBr}_{3}$ : (a) Cubic perovskite structure; $\left[\mathrm{PbBr}_{6}\right]^{4-}$ octahedra are indicated as shaded-gray surfaces, $\mathrm{Pb}^{2+}$ ions are indicated in black, $\mathrm{Br}^{-}$ions in red, and the $\mathrm{Cs}^{+}$cation in gold. (b) The orthorhombic lattice distortion (semitransparent lines) is compared to the parent cubic lattice (full lines). (c) Three-dimensional Brillouin zone of the cubic crystal lattice; (d) VB ARPES intensity as a function of energy, $E$ and in-plane momentum wave vectors, $k_{x}$ and $k_{y}$. The cubic and orthorhombic unit cells are indicated in red and black, respectively. (e),(f) Constant energy cuts of the ARPES intensity compared with DFT calculations for the cubic phase integrated over a range of $0.1 \AA^{-1}$ along the $k_{\perp}$ direction at the $\mathrm{VBM}[E=0 \mathrm{eV}$, (e)] and below the $\mathrm{VBM}[E=-1.1 \mathrm{eV},(\mathrm{f})]$. 
to orthorhombic. This is illustrated in the right half of Figs. 1(e) and 1(f), where the data is compared to DFT calculations for the cubic phase of $\mathrm{CsPbBr}_{3}$.

This is at odds with DFT calculations for the orthorhombic phase, which predicts the VBM at the $\bar{\Gamma}$ point $[30,60]$. To exclude matrix element effects and dispersion in the direction orthogonal to the sample surface $\left(k_{\perp}\right)$, we performed energy- and polarization-dependent ARPES measurements [30], which reveal no signature of an additional VBM at the $\bar{\Gamma}$ point. The observation of a larger $k$-space periodicity is not compatible with the scenario of a surface reconstruction. The additional potential associated with a periodic lattice distortion generally manifests itself with the appearance of backfolded bands and gaps opening at the novel Bragg planes. However, the spectral weight transfer to the novel bands is proportional to the strength of the perturbing potential and often hardly observable [61], e.g., for the methylammonium lead triiodide perovskite $\left(\mathrm{MAPbI}_{3}\right)$ [62,63], where no signatures of backfolded orthorhombic bands were observed by ARPES, despite a clear orthorhombic diffraction pattern. To calculate explicitly the spectral weight transfer upon the structural distortions, we follow a band unfolding procedure proposed by $\mathrm{Ku}$ et al. [64] and implemented in the Quantum ESPRESSO package [65]. The calculations confirm the absence of significant spectral weight transfer to the backfolded upper valence band in the orthorhombic phase [30]. For this reason we maintain the cubic phase notation for highsymmetry points throughout the text.

The material's band structure has been investigated as a function of the photon energy, and Fig. 2 shows the measured valence band at $33.5 \mathrm{eV}$, the VBM is found to correspond to $k_{\perp} \simeq 0.5 \AA^{-1}$, close to the cubic bulk $R$ point [30]. The data correspond to the band dispersion along the $\bar{\Gamma}-\bar{M}-\bar{X}-\bar{\Gamma}$ path of the surface Brillouin zone and are compared with the spectral weight of the orthorhombic bands unfolded on the cubic $X-R-M-X$ path. The upper valence band disperses for approximately $1.5 \mathrm{eV}$ below the VBM, before reaching a deeper valence manifold, where bands are not individually resolved.

Although in the room-temperature orthorhombic phase the ARPES spectral weight follows qualitatively the DFT bands for the cubic phase, the band dispersion is modified by the structural distortion. In fact, the effective mass computed for the orthorhombic phase is $0.17 \mathrm{~m}_{e}$, larger than the cubic phase mass of $0.12 m_{e}$ [30]. To determine the experimental hole effective mass, we turn to a quantitative analysis of the upper valence band dispersion which we compare with ab initio calculations for the orthorhombic structure. ARPES data along the $\bar{\Gamma}-\bar{M}$ direction are shown in Fig. 3. The VB energy distribution curves are well fitted by a Gaussian line shape whose width (which is not resolution limited) is likely determined by thermal broadening with possible contributions from disorder and orthogonal momentum dispersion. To determine $m_{\text {exp }}$,

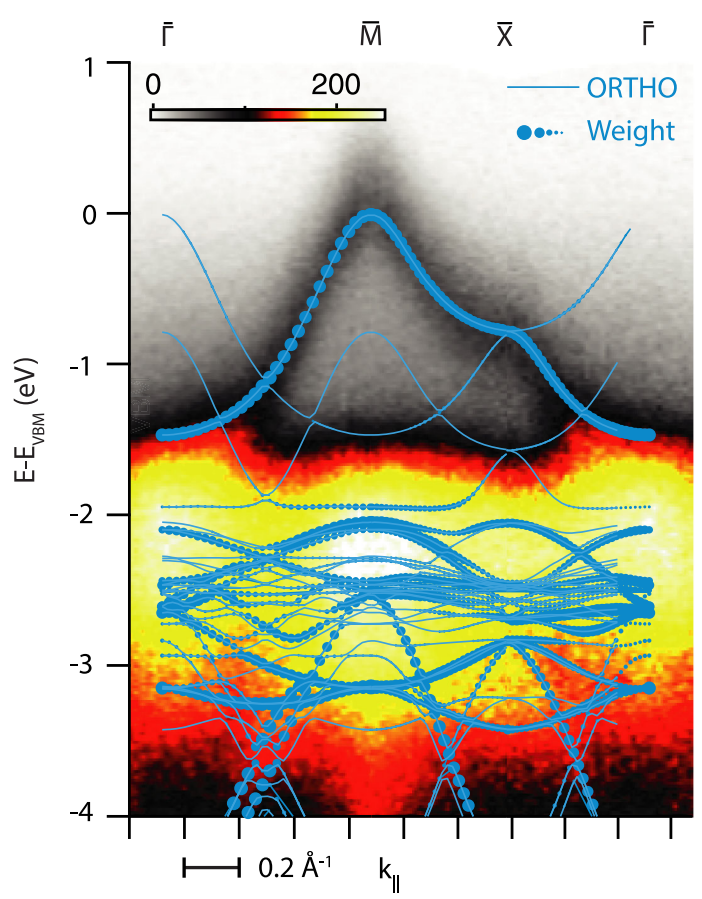

FIG. 2. Photoemission intensity as a function of energy and parallel momentum, along the path $\bar{\Gamma}-\bar{M}-\bar{X}-\bar{\Gamma}$. Cyan lines: Orthorhombic DFT bands. Markers: Orthorhombic band unfolding onto the cubic unit cell, the spectral weight is proportional to the marker size.

the valence band was fitted with a parabolic dispersion around the band maximum [30], the corresponding fit is shown in Fig. 3. The obtained value $m_{\exp }=0.26 \pm 0.02 m_{e}$ is in good agreement with optical measurements on $\mathrm{CsPbBr}_{3}$ [66], where a reduced exciton mass of $m_{\text {exc }}=$ $0.126 m_{e}$ was deduced, if one assumes balanced electron

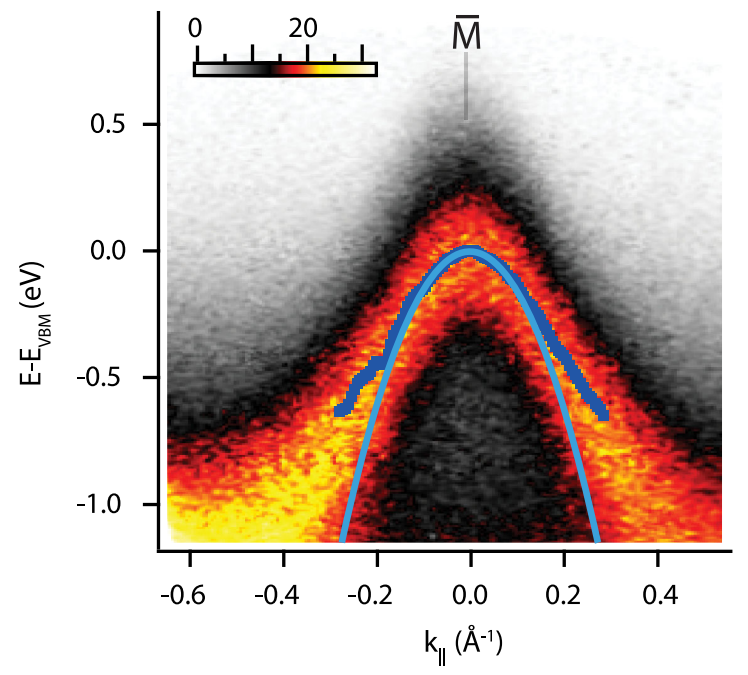

FIG. 3. ARPES intensity as a function of energy and parallel momentum showing the VBM along the $\bar{\Gamma}-\bar{M}-\bar{\Gamma}$ direction. The fitted band maxima are indicated as blue points. Cyan curve: Parabolic band fitted around the band maximum. 

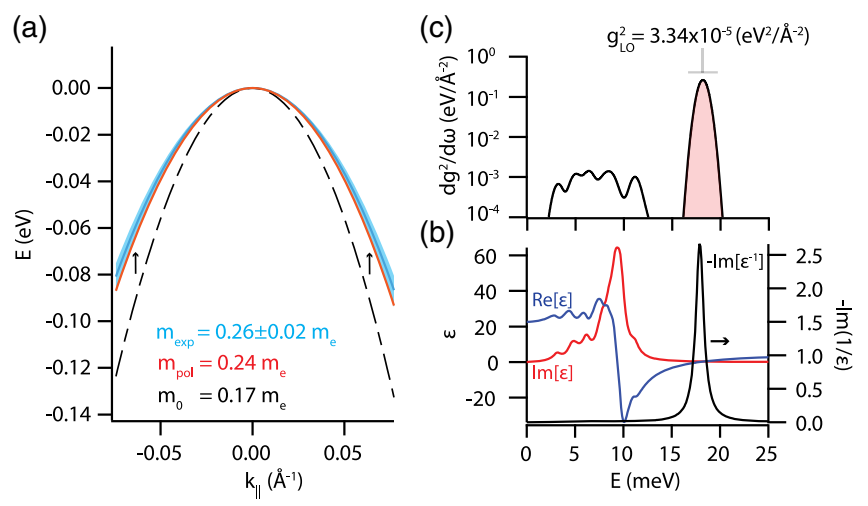

FIG. 4. (a) Comparison between the experimental dispersion ( $m_{\text {exp }}$, cyan line, the shaded area indicates the experimental uncertainty) and the theoretical effective mass $m_{0}$ computed from theory (black dot-dashed line). The renormalized mass including electron-phonon interaction $m_{\text {pol }}$ is plotted in red. (b) Computed dielectric function, real (blue line) and imaginary (red line) part are shown on the left-hand axis; the loss function $-\operatorname{Im}(1 / \epsilon)$ is plotted on the right-hand axis. (c) Logarithmic plot of the density of coupling $d\left(g^{2}\right) / d \omega$ to optical phonons [30], the shaded area indicates the integration region for determining the coupling constant $g_{\mathrm{LO}}^{2} \cdot d\left(g^{2}\right) / d \omega$ was broadened by convolution with a Gaussian function (1.2 meV FWHM) for clarity.

and hole effective masses, which appears justified by our DFT calculations.

The effective mass calculated at the HSE level of theory for the orthorhombic phase $\left(m_{0}=0.17 m_{e}\right)$ is compared to $m_{\text {exp }}$ in Fig. 4(a). Theory substantially underestimates $m_{\text {exp }}$, with an experimental mass enhancement of $\approx 50 \%$. Theoretical studies indicate an increase of the calculated effective mass for an increase of the computed band gap [11-13]: we studied the band gap and effective mass evolution in the orthorhombic phase using a PBE-based hybrid functional with increasing amounts of Fock exchange. In no circumstances, even by large overestimates of the band gap, do we observe a deviation of the effective mass larger than $10 \%$ [30]. To assess the performance of the HSE functional, we included $G_{0} W_{0}$ corrections to the cubic phase of the material. Our results compare well with previous studies $[8,67]$ and a comparison between HSE and $G_{0} W_{0}$ effective masses shows minor changes $(\approx 14 \%)$, indicating that the hybrid HSE functional gives a good description of the band structure [30]. Thermal lattice fluctuations can modify the average electronic properties of LHPs [68]. We calculate the effective mass change and the Rashba spin splitting upon lattice distortion along the phonon modes and conclude that these have low impact on the orthorhombic phase of $\mathrm{CsPbBr}_{3}$ [30]. A small Rashba parameter of $0.05 \mathrm{eV} \AA$ is reported for $\mathrm{CsPbBr}_{3}$ [69], which cannot induce an observable modification of the dispersion at room temperature [30]. These findings seem to rule out electronic correlations and thermal fluctuations as the main reasons for the mass renormalization observed.
An important mechanism, not accounted for by the DFT calculations and relevant for polar materials, is the interaction between the photogenerated hole and longitudinal optical phonons. ARPES is sensitive to many-body interactions, encoded in the single particle spectral function [70]. In particular, for polaronic systems, such interactions manifest themselves as a renormalization of the bare band dispersion and with the appearance of satellite peaks in the photoemission spectrum [71,72]. The satellites appear on the low-energy side of the main quasiparticle peak, at an energy separation corresponding to the relevant longitudinal optical (LO) phonon mode. In $\mathrm{CsPbBr}_{3}$ optical phonons have energies $\leq 25 \mathrm{meV}[73,74]$, and replicas cannot be resolved within the experimental linewidth. In contrast, our analysis of the quasiparticle dispersion captures the effective mass renormalization, which we attribute to electron-phonon interaction.

This interpretation is supported by recent theoretical predictions for $\mathrm{CsPbr}_{3}$ and related compounds, e.g., $\mathrm{MAPbI}_{3}$, which exhibits the same lattice structure and a similar phase diagram. Simulations of the electron-phonon interaction in $\mathrm{MAPbI}_{3}$ predict a mass enhancement of $\approx 30 \%$, where the interaction is dominated by coupling with longitudinal optical phonon modes, the most important being the $\mathrm{Pb}-\mathrm{I}$ stretching and bending modes, and the librational-translational modes of the methylammonium cation [45]. In the fully inorganic compound, where the latter modes are absent, simulations by Miyata et al. [15] show that the largest structural relaxation occurs on the $\mathrm{Pb}-\mathrm{Br}$ bond distance and on the $\mathrm{Pb}-\mathrm{Br}-\mathrm{Pb}$ bond angle, resulting in a reduction of the canting angle of the $\mathrm{PbBr}_{6}$ octahedra towards the undistorted cubic lattice.

To validate this picture, we estimated the Fröhlich electron-phonon interaction, following a method recently developed for polar semiconductors [45,59]. The Fröhlich vertex, which represents the matrix element for electron scattering by long-wavelength longitudinal optical phonons, can be written $[58,59]$ as

$$
g_{\nu}(\mathbf{q})=-i \frac{4 \pi e^{2}}{\Omega} \sum_{k} \sqrt{\frac{\hbar}{2 M_{k} \omega_{\mathbf{q} \nu}}} \frac{\hat{\mathbf{q}} \cdot Z_{k}^{*} \cdot \mathbf{e}_{k \nu}(\mathbf{q})}{\hat{\mathbf{q}} \cdot \varepsilon_{\infty} \cdot \hat{\mathbf{q}}}
$$

where $e$ is the electron charge, $\Omega$ is the volume of the unit cell, $M_{k}$ the mass of the atom $k, Z_{k}^{*}$ the Born effective charge tensor, $\varepsilon_{\infty}$ the high-frequency dielectric tensor, and $\omega_{\mathbf{q} \nu}$ and $\mathbf{e}_{k \nu}(\mathbf{q})$ the eigenvalue and eigenvector associated with the mode $\nu$ of momentum q. We computed the material's dielectric function, reported in Fig. 4(b), starting from $a b$ initio calculations of the phonon band structure of orthorhombic $\mathrm{CsPbBr}_{3}$ [30]. To assess the relative importance of the different phononic contributions in our calculations, the energy density of coupling $d\left(g^{2}\right) / d \omega$ [30] is plotted as a function of phonon energy in Fig. 4(c). The coupling is dominated by a maximum at an effective energy of $\hbar \tilde{\omega}_{\mathrm{LO}}=18.2 \mathrm{meV}$, in the energy region of $\mathrm{Pb}-\mathrm{Br}$ 
stretching modes $[13,15]$. The effective electron-phonon coupling to such modes is obtained integrating $d\left(g^{2}\right) / d \omega$ from 12 to $25 \mathrm{meV}$ [see Fig. 4(b)], resulting in $\tilde{g}_{\mathrm{LO}}^{2}=3.34 \times 10^{-5} \mathrm{eV}^{2} / \AA^{-2}$. Our calculation reveals that the coupling to the $\mathrm{Pb}-\mathrm{Br}$ stretching modes is 2 orders of magnitude stronger compared to modes appearing in the energy range between 2 and $13 \mathrm{meV}$ in Fig. 4(c), which can be associated with coupled stretching-bending modes of $\mathrm{Pb}-\mathrm{Br}[15]$.

Following these calculations, we proceed to estimate the mass renormalization from the Fröhlich model [57], valid for a parabolic band dispersion and coupling to a single dispersionless LO phonon mode. In this limit, it can be shown that the coupling matrix elements $g_{\nu}(\mathbf{q})$ reduce to the well-known Fröhlich coupling matrix elements [59]. The dimensionless Fröhlich coupling parameter, $\alpha$, can be expressed in terms of the $a b$ initio effective coupling strength $\tilde{g}_{\mathrm{LO}}^{2}$ as

$$
\alpha=\frac{\Omega}{4 \pi e^{2}} \frac{\tilde{g}_{\mathrm{LO}}^{2}}{\left(\hbar \tilde{\omega}_{\mathrm{LO}}\right)^{2}}\left(\frac{2 m_{0} \tilde{\omega}_{\mathrm{LO}}}{\hbar}\right)^{1 / 2},
$$

with $m_{0}$ the bare effective mass. We obtain $\alpha=1.81$, which fall into the weak to intermediate coupling regime. In this regime, the Feynman polaron model provides a good approximation for the quasiparticle mass $[45,75,76]$ :

$$
m_{\mathrm{pol}}=m_{0}\left(1+\frac{\alpha}{6}+0.025 \alpha^{2}+\cdots\right) .
$$

Here $m_{\mathrm{pol}}$ is the renormalized polaron mass, and $m_{0}$ is the bare quasiparticle mass extracted from our DFT calculations. The resulting $m_{\mathrm{pol}}=0.24 m_{e}$ is compared to the experimental result in Fig. 4. The result, in agreement with experiment within the experimental uncertainty, indicates that our model captures the main physics behind the hole quasiparticle dressing. Within the Feynman model, it is also possible to estimate the polaron binding energy and radius to be $34 \mathrm{meV}$ and $58 \AA$, respectively. Thus, the polaron resulting from an excess hole in $\mathrm{CsPbr}_{3}$ single crystals is large, extending over several lattice unit cells. We note that these simple estimates of polaronic radius and binding energy could be refined by a recently developed polaron model, capable of computing the polaron wave function ab initio [77]. Interestingly, in the case of $\mathrm{CsPbBr}_{3}$ nanocrystals, signatures of hole self-trapping were reported [78], suggesting that the electron-phonon interaction in LHPs' nanostructures may be altered $[79,80]$. The adopted theoretical method can be readily generalized to multiple coupled LO phonon modes [45], as in the case of hybrid organic-inorganic LHPs. Therefore, we expect it to be capable of predicting the carrier effective masses in the whole family of LHPs.

In conclusion, our work provides the first experimental reference for the momentum-resolved electronic structure of $\mathrm{CsPbr}_{3}$ in the orthorhombic phase. Fits of the electronic dispersion provide an experimental value for the effective mass $m_{\exp }=0.26 \pm 0.02 m_{e}$, which we found to exceed the theoretical result of $m_{0}=0.17 m_{e}$. The observed mass renormalization is ascribed to electronphonon interaction dominated by $\mathrm{Pb}-\mathrm{Br}$ stretching modes, responsible for the formation of large Fröhlich polarons. $A b$ initio electron-phonon coupling calculations are in quantitative agreement with the experiment, demonstrating that the employed theoretical method can correctly predict the carrier effective mass of LHPs from first principles. Our findings provide direct experimental evidence in the electronic structure that charge carriers in single-crystalline LHPs form large polarons and that the corresponding modification to the microscopic scattering rates must be taken into account to explain the exceptional transport properties of LHPs.

This work was supported by the ERC Advanced Grant No. 695197 (DYNAMOX), the Max-Planck-EPFL Center of Molecular Nanoscience and Technology, the German Research Foundation (DFG) through the Emmy Noether program under grant number RE 3977/1, and the Swiss National Science Foundation via the NCCR's MUST and MARVEL and Grants No. 200021-179139 and No. IZLRZ2-163919 and No. 206021-182994. MC thanks the Alexander von Humboldt Stiftung for support during his sabbatical at the Fritz Haber Institute. M. P., S. P, N. C., and A. C. wrote the manuscript; S. P. and M.P. analyzed the experimental data; S.P., M.P., R.P.X., and L.R. acquired the ARPES data; D. N.D., O. N., and M. V. K. grew and characterized the samples; N.C., R. d.G., and N. M. performed the numerical calculations; M. P., S.P., A. C., G. G., S. R., T.B., and L.P. provided technical support during the experiments; M.C. conceived and supervised the project, all authors contributed to the discussion and provided critical feedback to the manuscript.

*michele.puppin@epfl.ch majed.chergui@epfl.ch

[1] M. A. Green, E. D. Dunlop, D. H. Levi, J. Hohl-Ebinger, M. Yoshita, and A. W. Ho-Baillie, Solar cell efficiency tables (version 54), Prog. Photovoltaics 27, 565 (2019).

[2] L. N. Quan, F. P. G. d. Arquer, R.P. Sabatini, and E. H. Sargent, Perovskites for light emission, Adv. Mater. 30, 1801996 (2018).

[3] S. Yakunin, L. Protesescu, F. Krieg, M. I. Bodnarchuk, G. Nedelcu, M. Humer, G. De Luca, M. Fiebig, W. Heiss, and M. V. Kovalenko, Low-threshold amplified spontaneous emission and lasing from colloidal nanocrystals of caesium lead halide perovskites, Nat. Commun. 6, 8056 (2015).

[4] M. B. Johnston and L. M. Herz, Hybrid perovskites for photovoltaics: Charge-carrier recombination diffusion, and radiative efficiencies, Acc. Chem. Res. 49, 146 (2016). 
[5] L. M. Herz, Charge-carrier mobilities in metal halide perovskites: Fundamental mechanisms and limits, ACS Energy Lett. 2, 1539 (2017).

[6] J. S. Manser, J. A. Christians, and P. V. Kamat, Intriguing optoelectronic properties of metal halide perovskites, Chem. Rev. 116, 12956 (2016).

[7] D. A. Egger, A. Bera, D. Cahen, G. Hodes, T. Kirchartz, L. Kronik, R. Lovrincic, A. M. Rappe, D. R. Reichman, and O. Yaffe, What remains unexplained about the properties of halide perovskites?, Adv. Mater. 30, 1800691 (2018).

[8] M. A. Becker, R. Vaxenburg, G. Nedelcu, P. C. Sercel, A. Shabaev, M. J. Mehl, J. G. Michopoulos, S. G. Lambrakos, N. Bernstein, J. L. Lyons, T. Stöferle, R. F. Mahrt, M. V. Kovalenko, D. J. Norris, G. Rainò, and A. L. Efros, Bright triplet excitons in caesium lead halide perovskites, Nature (London) 553, 189 (2018).

[9] Y. Kang and S. Han, Intrinsic Carrier Mobility of Cesium Lead Halide Perovskites, Phys. Rev. Applied 10, 044013 (2018).

[10] X. Chen, D. Han, Y. Su, Q. Zeng, L. Liu, and D. Shen, Structural and electronic properties of inorganic mixed halide perovskites, Phys. status solidi (RRL)—Rapid Res. Lett. 12, 1800193 (2018).

[11] B. Traoré, G. Bouder, W. Lafargue-Dit-Hauret, X. Rocquefelte, C. Katan, F. Tran, and M. Kepenekian, Efficient and accurate calculation of band gaps of halide perovskites with the tran-blaha modified becke-johnson potential, Phys. Rev. B 99, 035139 (2019).

[12] Y. Kang and S. Han, Intrinsic Carrier Mobility of Cesium Lead Halide Perovskites, Phys. Rev. Applied 10, 044013 (2018).

[13] S. Poncé, M. Schlipf, and F. Giustino, Origin of low carrier mobilities in halide perovskites, ACS Energy Lett. 4, 456 (2019).

[14] Y. He, L. Matei, H. J. Jung, K. M. McCall, M. Chen, C. C. Stoumpos, Z. Liu, J. A. Peters, D. Y. Chung, B. W. Wessels, M. R. Wasielewski, V. P. Dravid, A. Burger, and M. G. Kanatzidis, High spectral resolution of gamma-rays at room temperature by perovskite $\mathrm{CsPbBr}_{3}$ single crystals, Nat. Commun. 9, 1609 (2018).

[15] K. Miyata, D. Meggiolaro, M. T. Trinh, P. P. Joshi, E. Mosconi, S. C. Jones, F. D. Angelis, and X.-Y. Zhu, Large polarons in lead halide perovskites, Sci. Adv. 3, e1701217 (2017).

[16] H. Fröhlich, H. Pelzer, and S. Zienau, Properties of slow electrons in polar materials, London, Edinburgh, and Dublin, Philos. Mag. J. Sci. 41, 221 (1950).

[17] X.-Y. Zhu and V. Podzorov, Charge carriers in hybrid organic-inorganic lead halide perovskites might be protected as large polarons, J. Phys. Chem. Lett. 6, 4758 (2015).

[18] H. Zhu, K. Miyata, Y. Fu, J. Wang, P. P. Joshi, D. Niesner, K. W. Williams, S. Jin, and X.-Y. Zhu, Screening in crystalline liquids protects energetic carriers in hybrid perovskites, Science 353, 1409 (2016).

[19] C. C. Stoumpos and M. G. Kanatzidis, The renaissance of halide perovskites and their evolution as emerging semiconductors, Acc. Chem. Res. 48, 2791 (2015).

[20] E. J. Juarez-Perez, R. S. Sanchez, L. Badia, G. GarciaBelmonte, Y.S. Kang, I. Mora-Sero, and J. Bisquert,
Photoinduced giant dielectric constant in lead halide perovskite solar cells, J. Phys. Chem. Lett. 5, 2390 (2014).

[21] S.-H. Turren-Cruz, A. Hagfeldt, and M. Saliba, Methylammonium-free, high-performance, and stable perovskite solar cells on a planar architecture, Science 362, 449 (2018).

[22] Z. Song, C. Wang, A. B. Phillips, C. R. Grice, D. Zhao, Y. Yu, C. Chen, C. Li, X. Yin, R. J. Ellingson, M. J. Heben, and Y. Yan, Probing the origins of photodegradation in organicinorganic metal halide perovskites with time-resolved mass spectrometry, Sustain. Energy Fuels 2, 2460 (2018).

[23] M. Kulbak, S. Gupta, N. Kedem, I. Levine, T. Bendikov, G. Hodes, and D. Cahen, Cesium enhances long-term stability of lead bromide perovskite-based solar cells, J. Phys. Chem. Lett. 7, 167 (2016).

[24] J. Liang, J. Liu, and Z. Jin, All-inorganic halide perovskites for optoelectronics: Progress and prospects, Solar RRL 1, 1700086 (2017).

[25] N. Calisi, S. Caporali, A. Milanesi, M. Innocenti, E. Salvietti, and U. Bardi, Composition-dependent degradation of hybrid and inorganic lead perovskites in ambient conditions, Top. Catal. 61, 1201 (2018).

[26] C. C. Stoumpos, C. D. Malliakas, J. A. Peters, Z. Liu, M. Sebastian, J. Im, T. C. Chasapis, A. C. Wibowo, D. Y. Chung, A. J. Freeman, B. W. Wessels, and M. G. Kanatzidis, Crystal growth of the perovskite semiconductor $\mathrm{CsPbBr}_{3}$ : A new material for high-energy radiation detection, Crystal Growth Des. 13, 2722 (2013).

[27] D. N. Dirin, I. Cherniukh, S. Yakunin, Y. Shynkarenko, and M. V. Kovalenko, Solution-grown $\mathrm{CsPbBr}_{3}$ perovskite single crystals for photon detection, Chem. Mater. 28, 8470 (2016).

[28] J. Ojeda, C. A. Arrell, J. Grilj, F. Frassetto, L. Mewes, H. Zhang, F. van Mourik, L. Poletto, and M. Chergui, Harmonium: A pulse preserving source of monochromatic extreme ultraviolet $(30-110 \mathrm{eV})$ radiation for ultrafast photoelectron spectroscopy of liquids, Struct. Dyn. 3, 023602 (2015).

[29] A. Crepaldi, S. Roth, G. Gatti, C. A. Arrell, J. Ojeda, F. van Mourik, P. Bugnon, A. Magrez, H. Berger, M. Chergui, and M. Grioni, Time-resolved ARPES at LACUS: Band structure and ultrafast electron dynamics of solids, CHIMIA Int. J. Chem. 71, 273 (2017).

[30] See Supplemental Material at http://link.aps.org/ supplemental/10.1103/PhysRevLett.124.206402 for details on the sample characterization, the ARPES experimental setup, additional energy-, polarization-, and temperaturedependent ARPES spectra, our fitting method and the computational details for DFT, band unfolding and $a b$ initio electron-phonon coupling calculations and the estimation of thermal effects, which includes Refs. [31-52].

[31] T. Komesu, X. Huang, T. R. Paudel, Y. B. Losovyj, X. Zhang, E. F. Schwier, Y. Kojima, M. Zheng, H. Iwasawa, K. Shimada, M. I. Saidaminov, D. Shi, A. L. Abdelhady, O. M. Bakr, S. Dong, E. Y. Tsymbal, and P. A. Dowben, Surface electronic structure of hybrid organo lead bromide perovskite single crystals, J. Phys. Chem. C 120, 21710 (2016).

[32] V. Strocov, Intrinsic accuracy in 3-dimensional photoemission band mapping, J. Electron Spectrosc. Relat. Phenom. 130, 65 (2003). 
[33] J. P. Perdew, K. Burke, and M. Ernzerhof, Generalized Gradient Approximation Made Simple, Phys. Rev. Lett. 77, 3865 (1996).

[34] D. R. Hamann, Optimized norm-conserving Vanderbilt pseudopotentials, Phys. Rev. B 88, 085117 (2013).

[35] M. Schlipf and F. Gygi, Optimization algorithm for the generation of ONCV pseudopotentials, Comput. Phys. Commun. 196, 36 (2015).

[36] A. Marini, C. Hogan, M. Grüning, and D. Varsano, yambo: An ab initio tool for excited state calculations, Comput. Phys. Commun. 180, 1392 (2009).

[37] D. Sangalli, A. Ferretti, H. Miranda, C. Attaccalite, I. Marri, E. Cannuccia, P. Melo, M. Marsili, F. Paleari, A. Marrazzo, G. Prandini, P. Bonfà, M. O. Atambo, F. Affinito, M. Palummo, A. Molina-Sánchez, C. Hogan, M. Grüning, D. Varsano, and A. Marini, Many-body perturbation theory calculations using the yambo code, J. Phys. Condens. Matter 31, 325902 (2019).

[38] N. Marzari, A. A. Mostofi, J. R. Yates, I. Souza, and D. Vanderbilt, Maximally localized Wannier functions: Theory and applications, Rev. Mod. Phys. 84, 1419 (2012).

[39] A. A. Mostofi, J. R. Yates, Y.-S. Lee, I. Souza, D. Vanderbilt, and N. Marzari, wannier90: A tool for obtaining maximallylocalised Wannier functions, Comput. Phys. Commun. 178, 685 (2008).

[40] A. A. Mostofi, J. R. Yates, G. Pizzi, Y.-S. Lee, I. Souza, D. Vanderbilt, and N. Marzari, An updated version of wannier90: A tool for obtaining maximally-localised Wannier functions, Comput. Phys. Commun. 185, 2309 (2014).

[41] J. B. Hoffman, A. L. Schleper, and P. V. Kamat, Transformation of sintered $\mathrm{CsPbBr}_{3}$ nanocrystals to cubic $\mathrm{CsPbI}_{3}$ and gradient $\mathrm{CsPbBr}_{x} \mathrm{I}_{3-x}$ through halide exchange, J. Am. Chem. Soc. 138, 8603 (2016).

[42] J. P. Perdew, M. Ernzerhof, and K. Burke, Rationale for mixing exact exchange with density functional approximations, J. Chem. Phys. 105, 9982 (1996).

[43] S. Baroni, S. de Gironcoli, A. Dal Corso, and P. Giannozzi, Phonons and related crystal properties from densityfunctional perturbation theory, Rev. Mod. Phys. 73, 515 (2001).

[44] X. Gonze and C. Lee, Dynamical matrices, Born effective charges, dielectric permittivity tensors, and interatomic force constants from density-functional perturbation theory, Phys. Rev. B 55, 10355 (1997).

[45] M. Schlipf, S. Poncé, and F. Giustino, Carrier Lifetimes and Polaronic Mass Enhancement in the Hybrid Halide Perovskite $\mathrm{CH}_{3} \mathrm{NH}_{3} \mathrm{Pbl}_{3}$ from Multiphonon Fröhlich Coupling, Phys. Rev. Lett. 121, 086402 (2018).

[46] R. X. Yang, J. M. Skelton, E. L. Da Silva, J. M. Frost, and A. Walsh, Spontaneous octahedral tilting in the cubic inorganic cesium halide perovskites $\mathrm{CsSnX}_{3}$ and $\mathrm{CsPbX}_{3}(\mathrm{X}=\mathrm{F}, \mathrm{Cl}$, Br, I), J. Phys. Chem. Lett. 8, 4720 (2017).

[47] M. Bokdam, T. Sander, A. Stroppa, S. Picozzi, D. Sarma, C. Franchini, and G. Kresse, Role of polar phonons in the photo excited state of metal halide perovskites, Sci. Rep. 6, 28618 (2016).

[48] F. Bertolotti, L. Protesescu, M. V. Kovalenko, S. Yakunin, A. Cervellino, S. J. Billinge, M. W. Terban, J. S. Pedersen, N. Masciocchi, and A. Guagliardi, Coherent nanotwins and dynamic disorder in cesium lead halide perovskite nanocrystals, ACS Nano 11, 3819 (2017).

[49] F. Zheng, L. Z. Tan, S. Liu, and A. M. Rappe, Rashba spinorbit coupling enhanced carrier lifetime in $\mathrm{CH}_{3} \mathrm{NH}_{3} \mathrm{PbI}_{3}$, Nano Lett. 15, 7794 (2015).

[50] K. Frohna, T. Deshpande, J. Harter, W. Peng, B. A. Barker, J. B. Neaton, S. G. Louie, O. M. Bakr, D. Hsieh, and M. Bernardi, Inversion symmetry and bulk Rashba effect in methylammonium lead iodide perovskite single crystals, Nat. Commun. 9, 1829 (2018).

[51] D. Niesner, M. Wilhelm, I. Levchuk, A. Osvet, S. Shrestha, M. Batentschuk, C. Brabec, and T. Fauster, Giant Rashba Splitting in $\mathrm{CH}_{3} \mathrm{NH}_{3} \mathrm{PbBr}_{3}$ Organic-Inorganic Perovskite, Phys. Rev. Lett. 117, 126401 (2016).

[52] A. D. Wright, C. Verdi, R. L. Milot, G. E. Eperon, M. A. Pérez-Osorio, H. J. Snaith, F. Giustino, M. B. Johnston, and L. M. Herz, Electron-phonon coupling in hybrid lead halide perovskites, Nat. Commun. 7, 11755 (2016).

[53] P. Giannozzi et al., QUANTUM ESPRESSO: A modular and open-source software project for quantum simulations of materials, J. Phys. Condens. Matter 21, 395502 (2009).

[54] P. Giannozzi et al., Advanced capabilities for materials modelling with Quantum ESPRESSO, J. Phys. Condens. Matter 29, 465901 (2017).

[55] J. Heyd, G. E. Scuseria, and M. Ernzerhof, Hybrid functionals based on a screened Coulomb potential, J. Chem. Phys. 118, 8207 (2003).

[56] J. Heyd, G. E. Scuseria, and M. Ernzerhof, Erratum: Hybrid functionals based on a screened coulomb potential, J. Chem. Phys. 124, 219906 (2006).

[57] H. Fröhlich, Electrons in lattice fields, Adv. Phys. 3, 325 (1954).

[58] P. Vogl, Microscopic theory of electron-phonon interaction in insulators or semiconductors, Phys. Rev. B 13, 694 (1976).

[59] C. Verdi and F. Giustino, Fröhlich Electron-Phonon Vertex from First Principles, Phys. Rev. Lett. 115, 176401 (2015).

[60] G. R. Yettapu, D. Talukdar, S. Sarkar, A. Swarnkar, A. Nag, P. Ghosh, and P. Mandal, Terahertz conductivity within colloidal $\mathrm{CsPbBr}_{3}$ perovskite nanocrystals: Remarkably high carrier mobilities and large diffusion lengths, Nano Lett. 16, 4838 (2016).

[61] J. Voit, L. Perfetti, F. Zwick, H. Berger, G. Margaritondo, G. Grüner, H. Höchst, and M. Grioni, Electronic structure of solids with competing periodic potentials, Science 290, 501 (2000).

[62] M.-I. Lee, A. Barragán, M. N. Nair, V. L. R. Jacques, D. L. Bolloc'h, P. Fertey, Khaoula Jemli, F. Lédée, G. TrippéAllard, E. Deleporte, A. Taleb-Ibrahimi, and A. Tejeda, First determination of the valence band dispersion of $\mathrm{CH}_{3} \mathrm{NH}_{3} \mathrm{PbI}_{3}$ hybrid organic-inorganic perovskite, J. Phys. D 50, 26LT02 (2017).

[63] J.-P. Yang, M. Meissner, T. Yamaguchi, X.-Y. Zhang, T. Ueba, L.-W. Cheng, S. Ideta, K. Tanaka, X.-H. Zeng, N. Ueno, and S. Kera, Band dispersion and hole effective mass of methylammonium lead iodide perovskite, Solar RRL 2, 1800132 (2018).

[64] W. Ku, T. Berlijn, and C.-C. Lee, Unfolding First-Principles Band Structures, Phys. Rev. Lett. 104, 216401 (2010). 
[65] F. Zheng, P. Zhang, and W. Duan, Quantum unfolding: A program for unfolding electronic energy bands of materials, Comput. Phys. Commun. 189, 213 (2015).

[66] Z. Yang, A. Surrente, K. Galkowski, A. Miyata, O. Portugall, R. J. Sutton, A. A. Haghighirad, H. J. Snaith, D. K. Maude, P. Plochocka, and R. J. Nicholas, Impact of the halide cage on the electronic properties of fully inorganic cesium lead halide perovskites, ACS Energy Lett. 2, 1621 (2017).

[67] J. Wiktor, U. Rothlisberger, and A. Pasquarello, Predictive determination of band gaps of inorganic halide perovskites, J. Phys. Chem. Lett. 8, 5507 (2017), PMID: 29077408.

[68] S. McKechnie, J. M. Frost, D. Pashov, P. Azarhoosh, A. Walsh, and M. Van Schilfgaarde, Dynamic symmetry breaking and spin splitting in metal halide perovskites, Phys. Rev. B 98, 085108 (2018).

[69] M. Isarov, L. Z. Tan, M. I. Bodnarchuk, M. V. Kovalenko, A. M. Rappe, and E. Lifshitz, Rashba effect in a single colloidal $\mathrm{CsPbr}_{3}$ perovskite nanocrystal detected by magneto-optical measurements, Nano Lett. 17, 5020 (2017).

[70] A. Damascelli, Probing the electronic structure of complex systems by ARPES, Phys. Scr., T109, 61 (2004).

[71] S. Moser, L. Moreschini, J. Jaćimović, O. S. Barišić, H. Berger, A. Magrez, Y. J. Chang, K. S. Kim, A. Bostwick, E. Rotenberg, L. Forró, and M. Grioni, Tunable Polaronic Conduction in Anatase $\mathrm{TiO}_{2}$, Phys. Rev. Lett. 110, 196403 (2013).

[72] Z. Wang et al., Tailoring the nature and strength of electronphonon interactions in the $\mathrm{SrTiO}_{3}(001)$ 2d electron liquid, Nat. Mater. 15, 835 (2016).

[73] D. M. Calistru, L. Mihut, S. Lefrant, and I. Baltog, Identification of the symmetry of phonon modes in $\mathrm{CsPbCl}_{3}$ in phase IV by raman and resonance-raman scattering, J. Appl. Phys. 82, 5391 (1997).

[74] P. Guo, Y. Xia, J. Gong, C. C. Stoumpos, K. M. McCall, G. C. Alexander, Z. Ma, H. Zhou, D. J. Gosztola, J. B. Ketterson et al., Polar fluctuations in metal halide perovskites uncovered by acoustic phonon anomalies, ACS Energy Lett. 2, 2463 (2017).

[75] R. P. Feynman, R. W. Hellwarth, C. K. Iddings, and P. M. Platzman, Mobility of slow electrons in a polar crystal, Phys. Rev. 127, 1004 (1962).

[76] J. T. Devreese and A. S. Alexandrov, Fröhlich polaron and bipolaron: Recent developments, Rep. Prog. Phys. 72, 066501 (2009).

[77] W. H. Sio, C. Verdi, S. Poncé, and F. Giustino, Polarons from First Principles, without Supercells, Phys. Rev. Lett. 122, 246403 (2019).

[78] F. G. Santomauro, J. Grilj, L. Mewes, G. Nedelcu, S. Yakunin, T. Rossi, G. Capano, A. Al Haddad, J. Budarz, D. Kinschel et al., Localized holes and delocalized electrons in photoexcited inorganic perovskites: Watching each atomic actor by picosecond x-ray absorption spectroscopy, Struct. Dyn. 4, 044002 (2017).

[79] A. J. Neukirch, W. Nie, J.-C. Blancon, K. Appavoo, H. Tsai, M. Y. Sfeir, C. Katan, L. Pedesseau, J. Even, J. J. Crochet et al., Polaron stabilization by cooperative lattice distortion and cation rotations in hybrid perovskite materials, Nano Lett. 16, 3809 (2016).

[80] C. M. Iaru, J. J. Geuchies, P. M. Koenraad, D. Vanmaekelbergh, and A. Y. Silov, Strong carrier-phonon coupling in lead halide perovskite nanocrystals, ACS Nano 11, 11024 (2017). 